\title{
A concept-wide association study to identify potential risk factors for nonadherence among prevalent users of antihypertensives
}

\author{
Karandeep Singh ${ }^{1}$ (D) । Niteesh K. Choudhry, ${ }^{2,3}$ | Alexis A. Krumme ${ }^{2}$ (D) । Caroline McKay ${ }^{4}$ । \\ Newell E. McElwee ${ }^{5}$ । Joe Kimura ${ }^{6}$ | Jessica M. Franklin ${ }^{2}$
}

${ }^{1}$ Departments of Learning Health Sciences and Internal Medicine, University of Michigan Medical School and University of Michigan School of Information, Ann Arbor, MI

${ }^{2}$ Division of Pharmacoepidemiology and Pharmacoeconomics, Department of Medicine, Brigham and Women's Hospital and Harvard Medical School, Boston, MA

${ }^{3}$ Center for Healthcare Delivery Sciences, Department of Medicine, Brigham and Women's Hospital and Harvard Medical School, Boston, MA

${ }^{4}$ Real World Value and Evidence, Janssen Scientific Affairs, LLC, Horsham, PA

${ }^{5}$ Health Economics and Outcomes, Boehringer Ingelheim, Ridgefield, CT

${ }^{6}$ Internal Medicine, Atrius Health and Harvard Medical School, Boston, MA

Correspondence

K. Singh, Departments of Learning Health Sciences and Internal Medicine University of Michigan Medical School and University of Michigan School of Information, $1161 \mathrm{H}$ NIB, 300 N. Ingalls St, Ann Arbor, MI 48109.

Email: kdpsingh@umich.edu

Funding information

National Institute of Diabetes and Digestive and Kidney Diseases, Grant/Award Number: 5K12DK111011; Merck

\begin{abstract}
Purpose: We sought to determine whether an association study using information contained in clinical notes could identify known and potentially novel risk factors for nonadherence to antihypertensive medications.

Methods: We conducted a retrospective concept-wide association study (CWAS) using clinical notes to identify potential risk factors for medication nonadherence, adjusting for age, sex, race, baseline blood pressure, estimated glomerular filtration rate, and a combined comorbidity score. Participants included Medicare beneficiaries 65 years and older receiving care at the Harvard Vanguard Medical Associates network from 2010-2012 and enrolled in a Medicare Advantage program. Concepts were extracted from clinical notes in the year prior to the index prescription date for each patient. We tested associations with the outcome for 5013 concepts extracted from clinical notes in a derivation cohort (4382 patients) and accounted for multiple hypothesis testing by using a false discovery rate threshold of less than $5 \%(q<.05)$. We then confirmed the associations in a validation cohort (3836 patients). Medication nonadherence was defined using a proportion of days covered (PDC) threshold less than 0.8 using pharmacy claims data.
\end{abstract}

Results: We found 415 concepts associated with nonadherence, which we organized into 11 clusters using a hierarchical clustering approach. Volume depletion and overload, assessment of needs at the point of discharge, mood disorders, neurological disorders, complex coordination of care, and documentation of noncompliance were some of the factors associated with nonadherence.

Conclusions: This approach was successful in identifying previously described and potentially new risk factors for antihypertensive nonadherence using the clinical narrative.

\section{KEYWORDS}

electronic health record, hypertension, medications, nonadherence, pharmacoepidemiology 


\section{1 | INTRODUCTION}

Adherence, defined as the extent to which patients take medications as prescribed ${ }^{1}$ is often poor for antihypertensive medications ${ }^{2}$ and is associated with worse health outcomes, including increased all-cause mortality, ${ }^{3}$ cardiovascular mortality, ${ }^{4,5}$ and stroke severity. ${ }^{6}$ A US survey of hypertensive adults found that $30.5 \%$ of respondents self-identified as being nonadherent to hypertensive medications. ${ }^{7}$ Other studies ${ }^{8,9}$ have reported the prevalence of nonadherence between $16 \%$ and $52 \%$, though the rates depend on how nonadherence is measured. ${ }^{10}$

Adherence is also a complex health behavior, and understanding the reasons why people do not consistently take prescribed medications has been a topic of intense study. Many systematic reviews have been conducted and have identified several factors that account for nonadherence. ${ }^{11-15}$ In one of these reviews, Krueger and colleagues ${ }^{13}$ grouped the reasons contributing to adherence behaviors into five categories: patient demographic-related factors (eg, low literacy), psychological and behavioral characteristics, treatment plan issues, disease-related issues (eg, severity of illness), and health care system issues (eg, relationship with provider and access to care). Psychological and behavioral characteristics linked to nonadherence include a belief that a medication is unimportant or harmful, depression, impaired cognitive function, forgetfulness, anger, stress, anxiety, and substance abuse. Treatment plan issues include the experience or fear of side effects, high price, inconvenience, and polypharmacy.

The existing body of literature has two limitations. First, the primary studies linking these factors to nonadherence rely on traditional epidemiological approaches, where a handful of possible exposures are linked to the outcome of nonadherence. Several other factors may be associated with nonadherence that have never been identified simply because they have not been studied. Second, the body of literature does not provide a way to determine which of these characteristics impact any given patient in a health care system without directly attempting to elicit this information from patients. For instance, a patient's prior experience with side effects may contribute to subsequent nonadherence, but determining which patients have experienced side effects is difficult on a large scale.

Though some health exposures that contribute to medication nonadherence may be found in administrative health data such as health insurance claims, many of the contributors are more complex and likely to be found only in the narrative of clinical notes and phone calls. Notes may provide a much richer picture, including symptoms, social issues, and life circumstances that could lead to problems with adherence. A comprehensive review of the electronic health record (EHR) is not feasible on a large scale, but automated extraction of concepts from clinical notes using natural language processing (NLP) software make such an endeavor feasible.

In this paper, we use a concept-wide association study ${ }^{16}$ (CWAS) to identify potential risk factors for medication nonadherence. We build off of prior work in which we demonstrated that data from the EHR can provide good predictions of future adherence. ${ }^{17}$ Unlike traditional epidemiologic studies that typically test a single association,

\section{KEY POINTS}

- We conducted a retrospective concept-wide association study using clinical notes to identify potential risk factors for medication nonadherence.

- We found 415 concepts associated with nonadherence after adjusting for age, sex, race, baseline blood pressure, estimated glomerular filtration rate, and a combined comorbidity score, which we organized into 11 clusters.

- Volume depletion and overload, assessment of needs at the point of discharge, mood disorders, neurological disorders, complex coordination of care, and documentation of noncompliance were some of the factors associated with nonadherence.

CWAS enables the discovery of new associations through a paradigm of simultaneous testing of multiple associations using pre-specified covariates. This paradigm was first established in the conduct of genome-wide association studies (GWAS) but has been used to discover associations between diseases and environmental exposures in environment-wide association studies (EWAS) ${ }^{18,19}$ and between a single genetic variant and multiple phenotypes using phenome-wide associations studies (PheWAS). ${ }^{20} \mathrm{~A}$ CWAS is useful to establish relationships between concepts documented in clinical documentation and health outcomes.

\section{2 | METHODS}

\section{1 | Data source}

Our data were drawn from a population of individuals 65 years and older enrolled in a Medicare Advantage program and receiving care at one of 24 practice sites of Harvard Vanguard Medical Associates (HVMA), a large multispecialty community-based medical group in eastern and central Massachusetts, between January 2010 and December 2012. For these individuals, we linked Medicare claims with structured and unstructured data in the EHR.

From claims data, we extracted data on demographics, all medical encounters, and diagnostic billing codes. In addition, we used medication refill data from pharmacy claims to measure adherence. The HVMA EHR data includes both structured fields, such as demographic characteristics and diagnostic billing codes, and unstructured fields, such as text from progress notes, electronic patient instructions, and patient letters, and telephone encounters (considered collectively as "clinical notes"). Because this network covers primary care, physician specialists, and laboratory testing, the EHR data cover nearly all of the patient's outpatient care encounters and procedures and contain longitudinal anthropometric and biomarker data. 
Prior to receipt and analysis by the research team, clinical notes were deidentified using several steps. Known identifiers such as patient and provider names were searched in the clinical notes and replaced with a series of random letters. All numbers were replaced with "1's. The remaining text was deidentified using the MITRE Identification Scrubber Toolkit (MIST), a machine-learning based deidentification tool with a demonstrated F-measure ${ }^{21}$ in excess of 0.9 .

\section{2 | Study design}

We conducted a retrospective cohort study to identify concepts in clinical notes that are associated with antihypertensive medication nonadherence. We then grouped the top concepts into clusters based on interconcept similarity.

We included outpatient adults 65 years or older who filled at least one prescription for an antihypertensive during 2011-2012 and were prevalent users of an antihypertensive medication. The first fill during this period prior to which patients also had a year of continuous insurance eligibility was considered the index fill. Prevalent users were defined as individuals who had at least one antihypertensive filled during the 365 days preceding the index date. We limited our analysis to prevalent users because the rates of nonadherence and factors influencing nonadherence are known to differ among new and prevalent users. ${ }^{22}$ Patients were excluded if they had fewer than 112 days of follow-up after the index fill, or if they had fewer than five notes in the year preceding the index date. We set a threshold of five notes based on prior experience. ${ }^{16}$ Patients with fewer than five notes are unlikely to have enough information in the EHR to allow accurate judgments about their exposures.

\section{3 | Outcome}

We defined the outcome of medication nonadherence based on the proportion of days covered (PDC) in the year following the index fill. PDC is defined as the proportion of days that the patient had medication available to him, based on a supply diary that strings together adjacent fills using the dates and days' supply of each pharmacy claim for an antihypertensive. We set a threshold of PDC $<0.8$ to define nonadherence based on prior research linking this threshold to improved cardiac outcomes and use in other quality measures. ${ }^{23,24}$ Since patients could fill more than one antihypertensive, we considered each antihypertensive class that the patient filled separately and averaged the PDC across classes, which is a valid way of measuring medication adherence. ${ }^{25}$

\subsection{Identification of concepts in clinical notes}

Concepts rather than individual words were extracted from clinical notes so that phrases representing the same idea could be grouped together when evaluating their association with the outcome (eg, "CHF" and "congestive heart failure"). Clinical notes consisted of all unstructured text available in the notes section of the EHR, including notes and phone calls from physicians of all specialties, nurses, case managers, and other care providers. The HVMA EHR is primarily an outpatient record and does not contain admission and inpatient notes. Concepts were coded as binary variables for each patient. Concepts were considered present if they were documented at least once in the clinical notes during the baseline period, and otherwise were considered absent.

Concepts were extracted from clinical notes in the year prior to the index prescription date for each patient (Figure 1) using the National Library of Medicine's MetaMap software ${ }^{26}$ (2014 version), which maps phrases to Unified Medical Language System (UMLS) codes known as concept unique identifiers (CUIs). Negated concepts were detected and removed using NegEx algorithm. ${ }^{27}$ Extracted concepts were restricted to the Systematized Nomenclature of MedicineClinical Terms (SNOMED-CT) ontology to limit mappings to clinically relevant concepts. Concepts were not limited by semantic type, so all types of concepts contained were extracted, including diagnoses, medications, signs and symptoms, exposures, geographical locations, and actions taken by a physician or patient. Mapping of phrases to multiple concepts was allowed. Concepts with less than $1 \%$ patient prevalence were not included in the analysis.

NLP systems may occasionally create erroneous mappings (eg, the word "Hi" in a phone call note maps to the concept for "Hawaii"). Instead of reporting the name of the concept intended by the UMLS definitions, we report the most common phrase for each concept (ie, we report "Hi" and not "Hawaii").

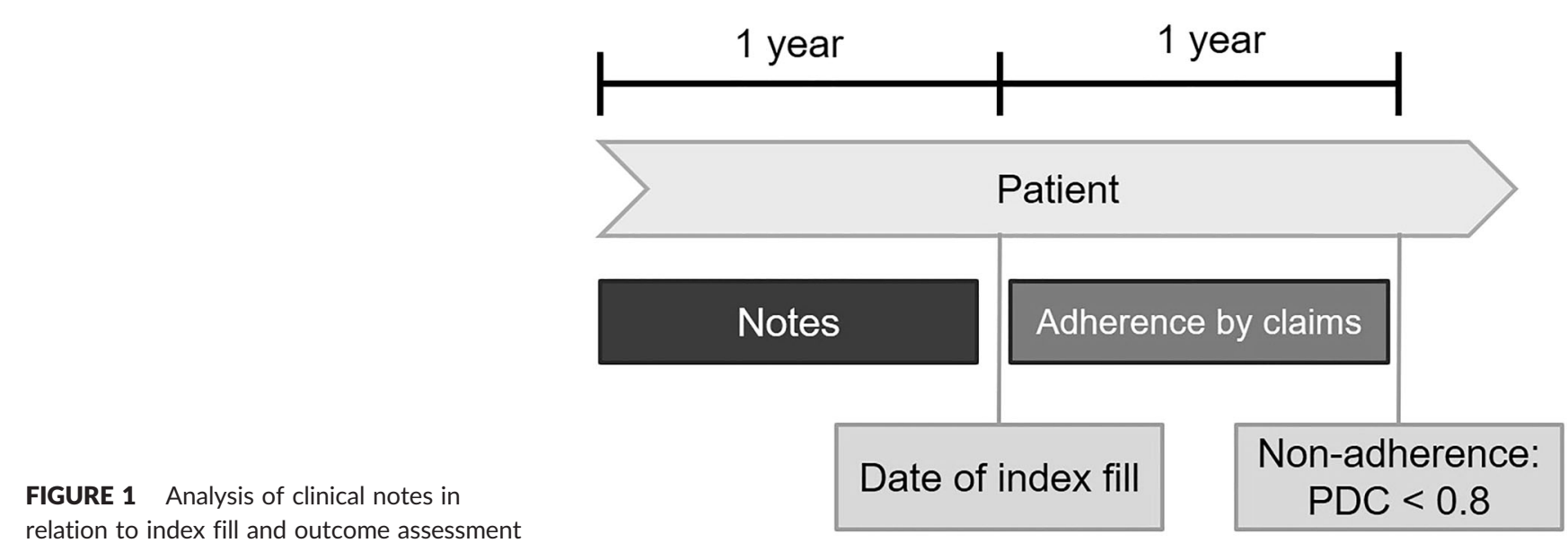

relation to index fill and outcome assessment 


\section{5 | Identification of covariates}

We selected covariates that may confound the relationship between the exposures and nonadherence, recognizing that these factors may in fact differ between the tested exposures. We defined baseline systolic and diastolic blood pressure and creatinine values for each lab test as the most recent result on or before the index date and estimated glomerular filtration rate (eGFR) was computed using the CKDEPI formula. ${ }^{28}$ The combined comorbidity score was computed using claims data in the year prior to the index date. ${ }^{29}$ We adjusted for comorbidity because we were concerned that sicker individuals may have lower rates of adherence due to inability to fill prescription. We selected the combined comorbidity score because it combines conditions from the Charlson and Elixhauser measures and was shown to have similar or slightly better performance in predicting mortality as compared with either of the individual comorbidity measures in a Medicare population similar to the population in our study. ${ }^{29}$

\subsection{Derivation and validation cohorts}

The overall cohort from which our derivation and validation cohorts were drawn consists of 24 primary care practices. We were concerned that practice patterns and nonadherence may differ between practices of different sizes. Thus, we stratified the assignment of patients to the derivation and validation cohorts at the practice level. Specifically, we ordered the practices based on their number of patients in our dataset. We then assigned practices to the derivation and validation set in alternating order.

\section{7 | Statistical analysis}

In the derivation cohort, multivariate logistic regression was performed to test the association for each of the concepts with medication nonadherence, adjusting for age, sex, black or Hispanic race, baseline systolic and diastolic blood pressure, estimated glomerular filtration rate, and a combined comorbidity score.

We accounted for multiple hypothesis testing using Storey method, which controls the false discovery rate, defined as the expected proportion of false positives among all significant hypotheses. $^{30,31}$ Using this method, $P$ values were transformed into $q$ values. Odds ratios (OR) and confidence intervals were not adjusted in any way. Concepts with $q$ values $<.05$ were reported as potential associations; this equates to a $5 \%$ expected proportion of false positives among all concepts declared to have associations. The false discovery rate method was chosen because of its many desirable properties ${ }^{30}$ : it explicitly controls the error rate of test conclusions among significant results, scales well in the face of increasing numbers of tests, and has increased power as compared to the Bonferroni method. After identifying potential associations in the derivation cohort, these were considered confirmed if the $P$ value in the validation cohort was less than .05 and the effect was in the same direction.
We clustered confirmed predictors into groups using several steps. First, we calculated pairwise phi correlation coefficients to measure similarity between concepts and then converted this to a distance measure by subtracting from 1 . Using this distance measure, we ran a hierarchical clustering algorithm with aggregation using complete linkage. Any number of clusters can be derived from the result of this algorithm (up to the number of observations) by "cutting" the hierarchical cluster tree at varying depths. We measured several cluster stability measures (Silhouette, point biserial correlation, CalinskiHarabasz, Davies-Bouldin, Ray-Turi, and Dunn) for all possible clustering solutions between five and 20 clusters. ${ }^{32}$ We selected an optimal number of clusters based on the cluster stability measures. We reviewed 10 randomly selected sentences for each concept and qualitatively assigned labels to each cluster.

TABLE 1 Baseline characteristics of the patients included in the study

\begin{tabular}{|c|c|c|c|}
\hline \multirow[b]{2}{*}{ Characteristic } & \multicolumn{2}{|c|}{ No. (\%) or Mean (SD) } & \multirow[b]{2}{*}{$\begin{array}{l}P \\
\text { Value }\end{array}$} \\
\hline & $\begin{array}{l}\text { Derivation cohort } \\
\text { (12 practices) }\end{array}$ & $\begin{array}{l}\text { Validation cohort } \\
\text { (12 practices) }\end{array}$ & \\
\hline Number of patients & 4382 & 3836 & \\
\hline Age, y & $76.6(5.4)$ & $76.0(5.4)$ & $<.001$ \\
\hline Sex & & & .005 \\
\hline Female & $2637(60.2 \%)$ & 2189 (57.1\%) & \\
\hline Male & 1745 (39.8\%) & 1647 (42.9\%) & \\
\hline Race & & & $<.001$ \\
\hline White & 3970 (90.6\%) & 3101 (80.8\%) & \\
\hline Black & 154 (3.5\%) & 359 (9.4\%) & \\
\hline Hispanic & $28(0.6 \%)$ & 42 (1.1\%) & \\
\hline Other/Unknown & $230(5.2 \%)$ & 335 (8.7\%) & \\
\hline $\begin{array}{l}\text { Baseline systolic blood } \\
\text { pressure, } \mathrm{mmHg}\end{array}$ & $130.3(14.8)$ & $130.0(14.5)$ & .366 \\
\hline $\begin{array}{l}\text { Baseline diastolic blood } \\
\text { pressure, } \mathrm{mmHg}\end{array}$ & $72.8(9.2)$ & $74.0(9.3)$ & $<.001$ \\
\hline $\begin{array}{l}\text { Baseline eGFR, } \\
\qquad \mathrm{mL} / \mathrm{min} / 1.73 \mathrm{~m}^{2}\end{array}$ & & & .001 \\
\hline$\geq 90$ & 480 (11.0\%) & 460 (12.0\%) & \\
\hline 60 to 89 & 2451 (55.9\%) & 2268 (59.1\%) & \\
\hline 30 to 59 & 1328 (30.3\%) & 1000 (26.1\%) & \\
\hline 15 to 29 & $104(2.4 \%)$ & $92(2.4 \%)$ & \\
\hline$<15$ & $19(0.4 \%)$ & $16(0.4 \%)$ & \\
\hline $\begin{array}{c}\text { Combined comorbidity } \\
\text { score, median (IQR) }\end{array}$ & $0(-1$ to 2$)$ & $0(-1$ to 2$)$ & .430 \\
\hline $\begin{array}{l}\text { Follow-up time after } \\
\text { index date, y, median } \\
\text { (IQR) }\end{array}$ & $360(360-360)$ & $360(360-360)$ & .763 \\
\hline $\begin{array}{l}\text { No. of notes in the year } \\
\text { prior to index date, } \\
\text { median (IQR) }\end{array}$ & $74(43-120)$ & 76.5 (47-118) & .015 \\
\hline
\end{tabular}

Abbreviation: eGFR, estimated glomerular filtration rate; IQR, interquartile range. 
Analyses were performed in R 3.3.2 (Vienna, Austria). q Values were computed using Storey's qvalue $\mathrm{R}$ package (available on Bioconductor). ${ }^{33}$ Hierarchical clustering was performed using the stats package and cluster stability metrics were computed using the clusterCrit package.

\section{3 | RESULTS}

We identified 8218 patients who met the inclusion and exclusion criteria (Table 1 and Figure 2) from 24 primary care practices, of whom 2088 (25.4\%) were nonadherent to antihypertensives (PDC < 0.8). We processed 770353 notes and extracted 32693 nonnegated concepts from clinical notes. We removed duplicate phrases and considered only the 5031 concepts with a prevalence of greater than or equal to $1 \%$. The median follow-up period during which we assessed the PDC was 360 days. We assigned 4382 patients to the derivation cohort and 3836 to the validation cohort based on their assigned primary care practice.

Using a false discovery rate threshold of less than $5 \%(q<.05)$, we identified 594 concepts significantly associated with adherence in the derivation cohort, 583 with nonadherence $(O R>1)$ and 11 with favorable adherence (OR < 1). Of these, 415 concepts had confirmed associations in the validation cohort based on $P$ value $<.05$ and concordant $\mathrm{OR}$ in the two cohorts. All validated concepts were associated with nonadherence $(O R>1)$. Based on several cluster quality measures (Table S1), we grouped the confirmed associations into 11 clusters (Table 2). The OR, confidence intervals, and $q$ values for individual concepts are provided in Table S2. Ten randomly selected sentences from which the cluster descriptions were derived are provided in Appendix S1.

Cluster 1 includes concepts related to volume depletion (eg, hypotension, IV fluids, dehydration, tachycardia, hydration, lightheadedness, and dry) and volume overload (eg, 1 pitting edema, pedal edema, lower extremity edema, BNP [beta natriuretic peptide], low salt diet, diuretic, CXR [chest X-ray], and weight gain). Additional concepts in cluster 1 relate to the evaluation of diagnoses that may mimic volume depletion (eg, WBC [white blood cell count] and urine culture to work-up infection) or volume overload (eg, DVT [deep venous thrombosis]). Cluster 2 broadly relates to case management (eg, Case Manager, Case Management, and nurse case manager), assessment of needs at discharge (eg, mobility in home, skilled nursing facility, medication teaching, assistive device, walker, discharged home, plan of care, HOME ASSESSMENT, rehab, commode, home exercise program, hospice), mood disorders (eg, mental illness, Anxiety/depression, and SSRI [selective serotonin reuptake inhibitor]), and social determinants of health (eg, Lives with spouse, family support, Social support, upset, afraid, and compliance). Cluster 3 captures neurological disorders. Cluster 4 includes items related to coordination of care between the physician's office and the patient (eg, "back" refers to voicemails left for patients asking them to call back, "Hi" refers to greetings in messages between care providers, "FW" refers to messages forwarded between care providers, "pls" is used as shorthand for "please" in the commonly used phrase "pls call patient," "letter" refers to letters written to patients, "Pool" refers to the pool of staff who answer phone calls for patients, and "adv" is shorthand for patients being "advised") and documentation of a variety of symptoms. Cluster 5 is focused on place of residence and related needs (eg, "hospital bed" in the context of use at home, nursing home, wheelchair, "assisted" used in the context of assisted living), refractoriness to treatment (eg, refractory, "unresponsive" used in the context of unresponsiveness to treatment), and noncompliance. Cluster 6 refers to management of cardiac arrhythmias with warfarin (eg, "spontaneous" used in the context of spontaneous development of palpitations/arrhythmias, "prothrombin time" linked to use of warfarin, "accident" includes a note to patients taking warfarin on what to do if they are in a car accident, and "remind" includes reminders to patients

FIGURE 2 Flow diagram of patient selection for study cohort

Excluded patients with no baseline creatinine

Excluded new users of anti-hypertensives and individuals with no available comorbidity information

Excluded patients without an HVMA primary care visit 8,274

Excluded patients no baseline blood pressure

8,271

Excluded patients with fewer than 5 notes

Final study cohort 


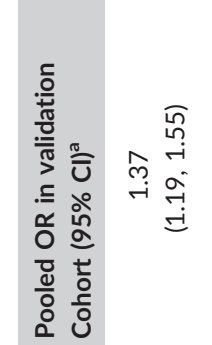

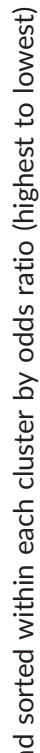

迹

章

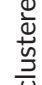

ป

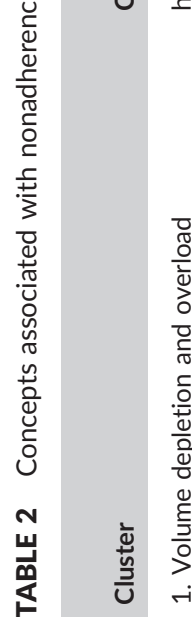

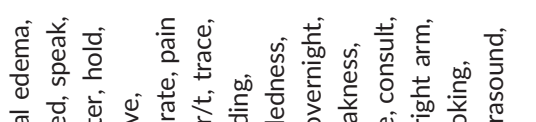

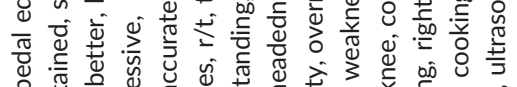

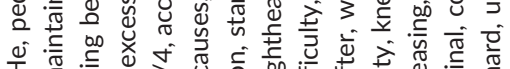

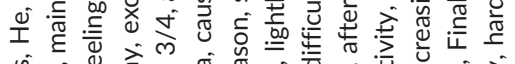

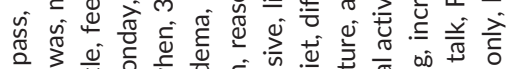

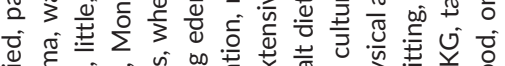

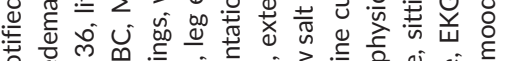

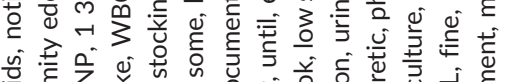

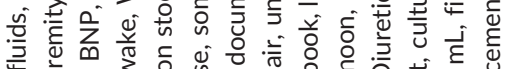

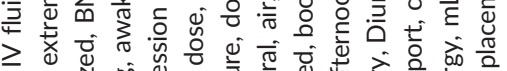

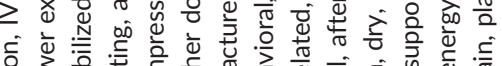

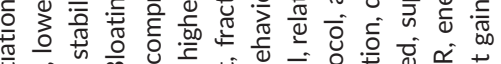

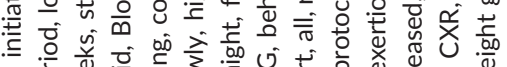

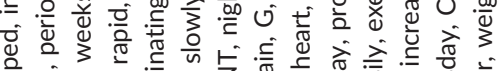

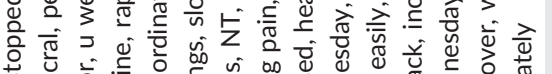

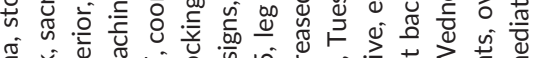

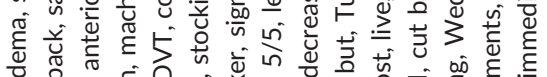

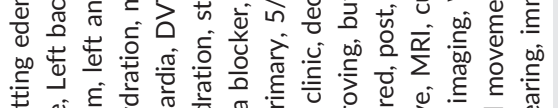

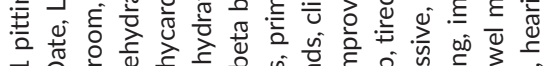

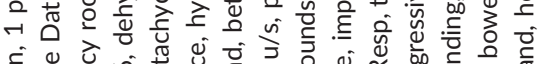

ص

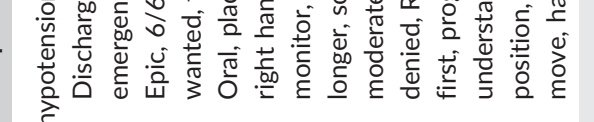

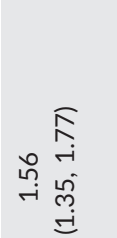

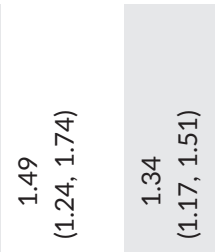

חָּ

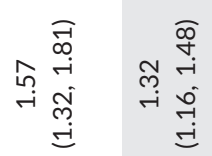

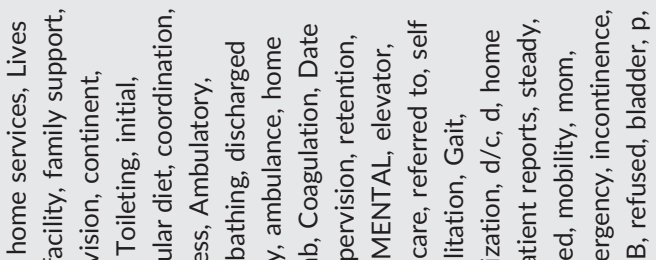

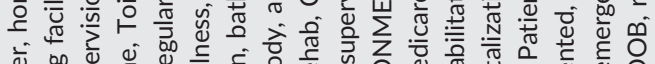

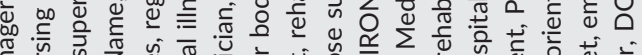

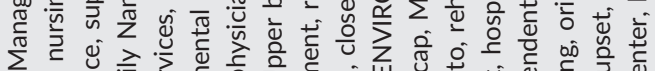

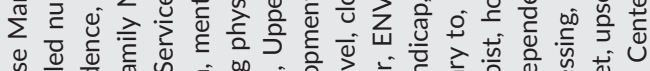

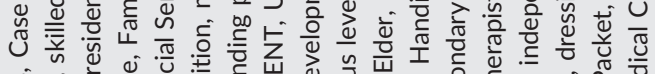

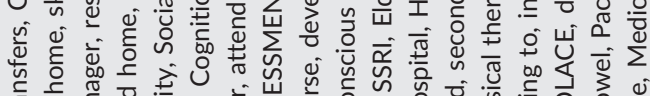

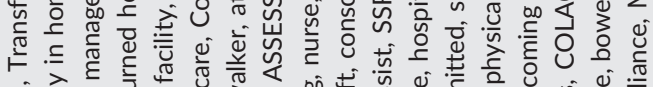

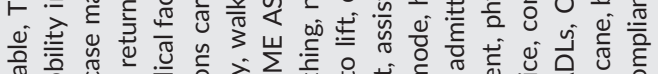

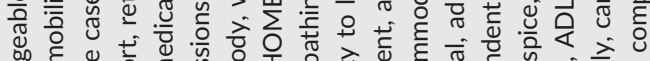

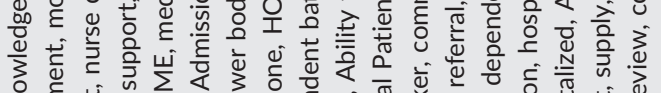

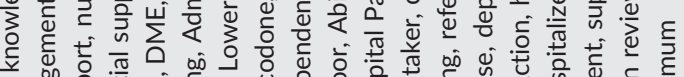

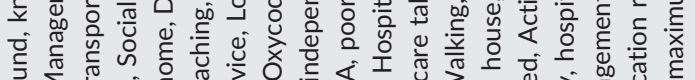

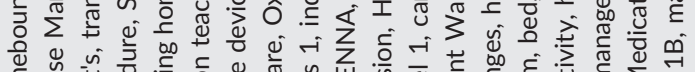
है

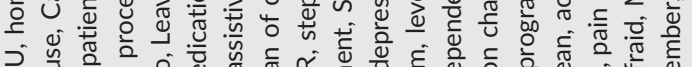

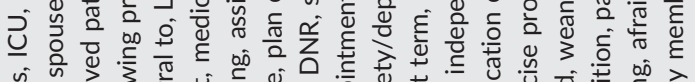

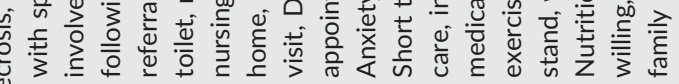

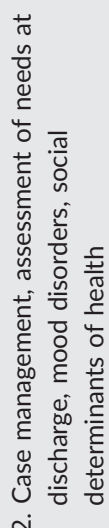

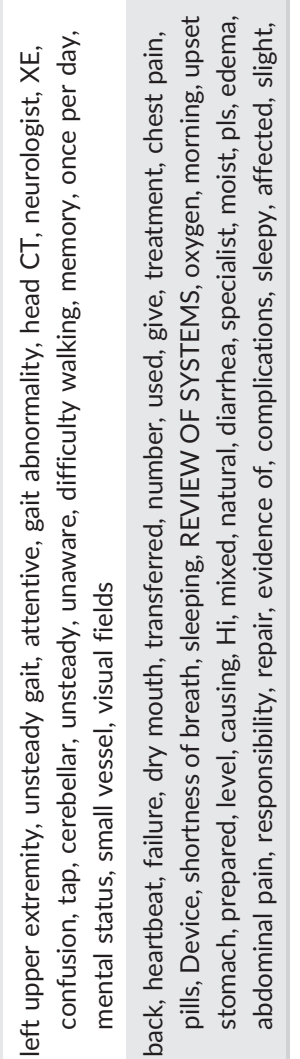

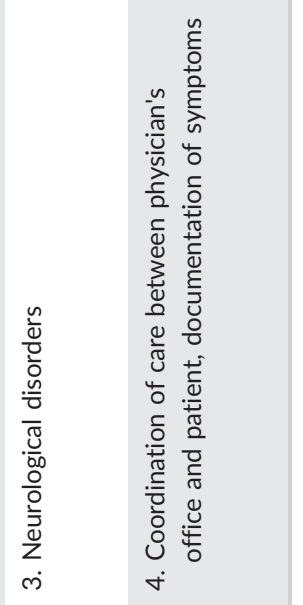




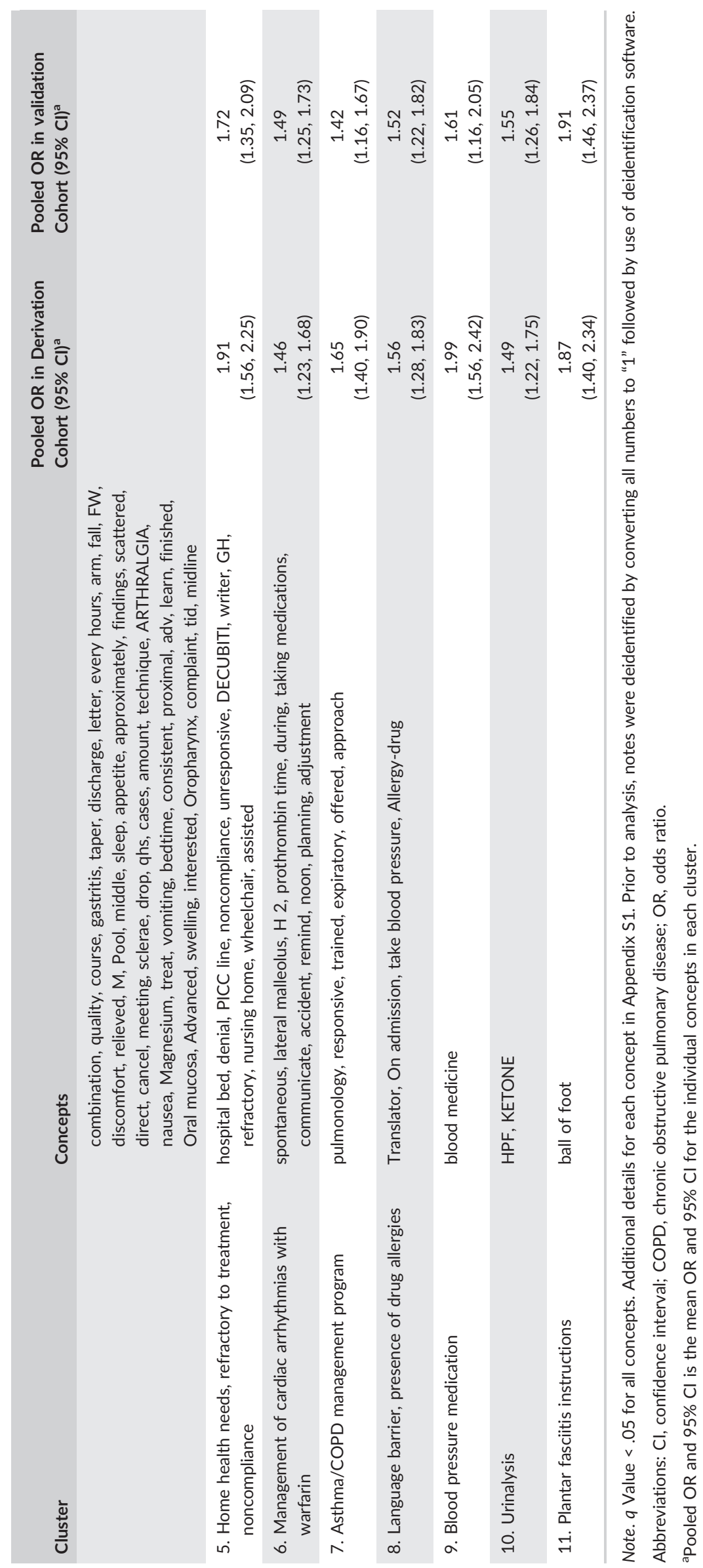


about the risks of warfarin and need for close monitoring). Cluster 7 refers to patients offered or enrolled in either the Asthma Management Program or COPD Management Program (eg, "pulmonology" refers to upcoming appointment with pulmonologist, "trained" includes references to "specially trained nurses" that comes from an invitation letter for the Asthma/COPD Management Program, and "expiratory" refers to increased expiratory time). Cluster 8 includes need for a translator due to a language barrier and allergies to medications. Cluster 9 refers to patients taking "blood pressure medicine." Cluster 10 relates to counting of cells per high power field (HPF) on urinalysis and measurement of ketones. Cluster 11 refers to language taken from plantar fasciitis patient instructions.

The 11 clusters we identified include several factors previously identified by the literature. Our analysis found supporting evidence for eight of the 12 risk factors for medication nonadherence described in a widely cited review article ${ }^{1}$ by Osterberg and Blaschke (Table 3). Krueger and colleagues ${ }^{13}$ performed a systematic review that classified factors affecting adherence into six categories: patient demographics, family/cultural issues, psychosocial and behavioral characteristics, treatment plan issues, disease-related issues, and health care system issues. We found supportive evidence for five of these: family/cultural issues (cluster 2), psychological and behavioral factors (cluster 2), treatment plan issues (clusters 5-7 and 9), diseaserelated issues (cluster 1), and health care system-related issues (clusters 2 and 4). We did not find any clusters related to patient demographics, but this is not surprising as we adjusted for demographic characteristics in our analysis.

\section{4 | DISCUSSION}

This is the first study to use a multiple hypothesis-testing approach utilizing text from the clinical notes to identify potential risk factors for medication nonadherence. Our findings mostly confirm existing knowledge on medication adherence. Our results are important for two reasons. First, existing knowledge has been drawn from multiple clinical studies using carefully assessed exposures and outcomes. That we were able to partially replicate the findings from published literature in a single retrospective cohort study using clinical notes and multiple hypothesis testing is promising because this approach may be useful for evaluating other clinical questions where the published literature is not as rich. Second, the concepts we identified can be directly used in identifying patients at risk for nonadherence. While Osterberg and Blaschke's review identified the presence of psychological problems as a risk factor for nonadherence, our study provides a mechanism for identifying such patients. Searching the notes for the phrases "Anxiety/depression," "mental illness," "SSRI," and "mood" may be effective ways of identifying such patients. Linking these phrases to clinical decision support would provide a means to flag high-risk patients for targeted interventions.

We also identified some surprising associations. We found that the phrase "Medicare" is linked to nonadherence. While the phrase would seem to imply that a patient is covered by Medicare, a review of the sample sentences (Appendix S1) reveals that the concept is often mentioned in the context of a Medicare notice of noncoverage, which is delivered to patients as they near completion of physical rehabilitation. We found the phrase "He" to be linked to nonadherence, which appears to be used in the clinical documentation to describe male patients (Appendix S1). Since we adjusted for sex in our analysis, its significance is likely explained by other contexts in which it was used beyond what we found in our review of sample sentences, as it was found in over $80 \%$ of patients. Krueger and colleagues' systematic review found male providers to be linked to favorable adherence, so the use of "he" to describe male providers would not appear to explain this association either. One other surprising finding was that the phrases "Lives with spouse," "Social support," and "family member" were all associated with nonadherence, contrary to previous literature identified by Krueger and colleagues. It is possible that clinicians are more likely to assess and document a patient's living situation if they deem the patient at greater risk

TABLE 3 Comparing predictors identified by a review article $^{1}$ to our notes-driven approach

\begin{tabular}{|c|c|c|}
\hline Predictors in Review Article & Related Phrases Identified Using a Notes-Driven Approach & Clusters \\
\hline Presence of psychological problems, particularly depression & mental illness, Anxiety/depression, SSRI, mood & 1,2 \\
\hline Presence of cognitive impairment & Cognition, confusion, memory, mental status & 2,3 \\
\hline Treatment of asymptomatic disease & -- & -- \\
\hline Inadequate follow-up or discharge planning & -- & -- \\
\hline Side effects of medication & $\begin{array}{l}\text { Hypotension, dehydration, tachycardia, beta blocker, Diuretic, } \\
\text { dry mouth, Allergy-drug }\end{array}$ & $1,4,8$ \\
\hline Patient's lack of belief in benefit of treatment & refused & 2 \\
\hline Patient's lack of insight into the illness & unaware & 3 \\
\hline Poor provider-patient relationship & -- & -- \\
\hline Presence of barriers to care or medications & upset, afraid, noncompliance, Translator & $2,5,8$ \\
\hline Missed appointments & cancel & 4 \\
\hline Complexity of treatment & medication teaching, Medication review & 2 \\
\hline Cost of medication, copayment, both & -- & -- \\
\hline
\end{tabular}


for nonadherence. Lastly, the relationship between clusters 9-11 and nonadherence is not clear upon review of the randomly sampled sentences (Appendix S1).

The rising adoption of EHRs and more recent development of data-sharing research networks creates an opportunity to systematically discover predictors of health outcomes from EHRs. We believe that this approach may be useful especially for the study of rare and understudied health outcomes and behaviors, where a systematic review of the literature may not be as fruitful.

\section{1 | Limitations}

As our approach is intended to be hypothesis generating, caution is needed when interpreting the results because the concepts identified as predictors may not be used consistently in notes despite evaluation of example sentences, may represent erroneous mapping by NLP software, may be confounded, or may represent false positive results (due to $5 \%$ false discovery rate). When concepts have multiple meanings or contexts in the notes, we cannot be certain which of these is responsible for the overall association with medication nonadherence, and this may introduce error and limit the interpretability of the analysis. Confounding and exposure misclassification can be particularly difficult to identify in this type of analysis. For example, the association between "COLACE" (cluster 2) and nonadherence may be confounded by "Oxycodone" (also in cluster 2) as stool softeners are commonly prescribed for prevention of opioid-induced constipation. This study draws from a relatively geographically and demographically homogenous population with a low fraction of non-white patients, so its findings may not generalize to other populations. Additionally, our study was limited by residual confounding. The causal mechanisms are likely to differ among the multiple exposures tested in this study, and thus, the covariates included in our analysis may not fully account for potentially confounders across the breadth of tested exposures.

\section{5 | CONCLUSIONS}

This approach was successful in identifying previously described and potentially new predictors of antihypertensive nonadherence using the clinical narrative as a by-product of routine care delivery.

\section{ETHICS STATEMENT}

The Partners Healthcare institutional review board approved this study, and the need for informed consent was waived.

\section{ACKNOWLEDGEMENT}

We are grateful to Angela Tong for her assistance with programming and data management.

\section{CONFLICT OF INTEREST}

N.K.C. reports grants from CVS Caremark during the conduct of the study and from Sanofi; AstraZeneca; Medisafe; the National Heart,
Lung, and Blood Institute; Merck; and Pharmaceutical Research and Manufacturers of America. N.K.C. and J.M.F. are coprincipal investigators with funding from Merck on this study. C.M. is a former employee of Merck and owns stock in the company. C.M. is a current employee of Janssen, a relationship that began after the study ended. N.E.M. is a former employee of Pfizer and Merck and owns stock in both companies. N.E.M. is a current employee of Boehringer Ingelheim, a relationship that began after this study ended. J.K. is an employee of Atrius Health, where the study was conducted. K.S. and A.A.K. have no relevant disclosures to report.

\section{FUNDING INFORMATION}

The research was supported by funding from Merck and by grant 5K12DK111011 from the National Institute of Diabetes and Digestive and Kidney Diseases on which Karandeep Singh is an appointee.

\section{AUTHOR CONTRIBUTION}

Study concept and design: Singh, Franklin, Choudhry, McKay, McElwee, and Kimura; Acquisition of data: Kimura, Krumme, Franklin, and Choudhry; Analysis and interpretation of data: Singh, Franklin, Choudhry, and McKay; Drafting of the manuscript: Singh and Franklin; Critical revision of the manuscript for important intellectual content: Singh, Krumme, Franklin, Choudhry, McKay, McElwee, and Kimura; Administrative, technical, or material support: Tong; Study supervision: Franklin.

\section{ORCID}

Karandeep Singh (D) https://orcid.org/0000-0001-8980-2330

Alexis A. Krumme (1) https://orcid.org/0000-0001-9633-861X

Jessica M. Franklin (D) https://orcid.org/0000-0002-8941-4116

\section{REFERENCES}

1. Osterberg L, Blaschke T. Adherence to medication. N Engl J Med. 2005;353(5):487-497. https://doi.org/10.1056/NEJMra050100

2. Cohen DL, Townsend RR. Medication non-adherence: a bigger problem than physicians assume. J Clin Hypertens (Greenwich). May 2016;18(9):949-950. https://doi.org/10.1111/jch.12838

3. Molnar MZ, Gosmanova EO, Sumida K, et al. Predialysis cardiovascular disease medication adherence and mortality after transition to dialysis. Am J Kidney Dis. 2016;68(4):609-618. https://doi.org/10.1053/j. ajkd.2016.02.051

4. Herttua K, Martikainen P, Batty GD, Kivimäki M. Poor adherence to statin and antihypertensive therapies as risk factors for fatal stroke. $J$ Am Coll Cardiol. 2016;67(13):1507-1515. https://doi.org/10.1016/j. jacc.2016.01.044

5. Kim S, Shin DW, Yun JM, et al. Medication adherence and the risk of cardiovascular mortality and hospitalization among patients with newly prescribed antihypertensive medications. Hypertension. 2016;67(3): 506-512. https://doi.org/10.1161/HYPERTENSIONAHA.115.06731

6. Lee KB, Lee J-Y, Choi N, et al. Association between insufficient medication of antihypertensives and the severity of acute ischemic stroke. Clin Hypertens. 2015;22(1):11. https://doi.org/10.1186/s40885-0160047-8

7. Tong X, Chu EK, Fang J, Wall HK, Ayala C. Nonadherence to antihypertensive medication among hypertensive adults in the United 
States-HealthStyles, 2010. J Clin Hypertens (Greenwich). 2016; 18(9):892-900. https://doi.org/10.1111/jch.12786

8. Schmieder RE, Ott C, Schmid A, et al. Adherence to antihypertensive medication in treatment-resistant hypertension undergoing renal denervation. J Am Heart Assoc. 2016;5(2). https://doi.org/10.1161/ JAHA.115.002343

9. Van Wijk BL, Klungel OH, Heerdink ER, de Boer A. Rate and determinants of 10-year persistence with antihypertensive drugs. J Hypertens. 2005;23(11):2101-2107.

10. Maeng DD, Snyder RC, Medico CJ, Mold WM, Maneval JE. Unused medications and disposal patterns at home: findings from a Medicare patient survey and claims data. J Am Pharm Assoc (2003). 2016; 56(1):41-46.e6. https://doi.org/10.1016/j.japh.2015.11.006

11. Gellad WF, Grenard J, McGlynn EA. A Review of Barriers to Medication Adherence: A Framework for Driving Policy Options. 2009.

12. Gellad WF, Grenard JL, Marcum ZA. A systematic review of barriers to medication adherence in the elderly: looking beyond cost and regimen complexity. Am J Geriatr Pharmacother. 2011;9(1):11-23. https://doi. org/10.1016/j.amjopharm.2011.02.004

13. Krueger KP, Berger BA, Felkey B. Medication adherence and persistence: a comprehensive review. Adv Ther. 2005;22(4):313-356.

14. Bowry ADK, Shrank WH, Lee JL, Stedman M, Choudhry NK. A systematic review of adherence to cardiovascular medications in resourcelimited settings. J Gen Intern Med. 2011;26(12):1479-1491. https:// doi.org/10.1007/s11606-011-1825-3

15. Marshall IJ, Wolfe CDA, McKevitt C. Lay perspectives on hypertension and drug adherence: systematic review of qualitative research. BMJ. 2012;345(1):e3953.

16. Singh K, Betensky RA, Wright A, Curhan GC, Bates DW, Waikar SS. A concept-wide association study of clinical notes to discover new predictors of kidney failure. Clin J Am Soc Nephrol. 2016;11(12): 2150-2158. https://doi.org/10.2215/CJN.02420316

17. Franklin JM, Gopalakrishnan C, Krumme AA, et al. The relative benefits of claims and electronic health record data for predicting medication adherence trajectory. Am Heart J. 2018;197:153-162. https://doi.org/ 10.1016/j.ahj.2017.09.019

18. Patel CJ, Bhattacharya J, Butte AJ. An environment-wide association study (EWAS) on type 2 diabetes mellitus. PLOS ONE. 2010;5(5): e10746. https://doi.org/10.1371/journal.pone.0010746

19. Patel $\mathrm{CJ}$, loannidis JPA. Studying the elusive environment in large scale. JAMA. 2014;311(21):2173-2174. https://doi.org/10.1001/jama.2014. 4129

20. Denny JC, Bastarache L, Ritchie MD, et al. Systematic comparison of phenome-wide association study of electronic medical record data and genome-wide association study data. Nat Biotechnol. 2013;31(12): 1102-1111. https://doi.org/10.1038/nbt.2749

21. Deleger L, Molnar K, Savova G, et al. Large-scale evaluation of automated clinical note de-identification and its impact on information extraction. J Am Med Inform Assoc. 2013;20(1):84-94. https://doi.org/ 10.1136/amiajnl-2012-001012

22. Blackburn DF, Swidrovich J, Lemstra M. Non-adherence in type 2 diabetes: practical considerations for interpreting the literature. Patient Prefer Adherence. 2013;7:183-189. https://doi.org/10.2147/PPA.S30613
23. Choudhry NK, Glynn RJ, Avorn J, et al. Untangling the relationship between medication adherence and post-myocardial infarction outcomes. Am Heart J. 2014;167(1):51-58.e5. https://doi.org/10.1016/j. ahj.2013.09.014

24. Trends in Part C \& D Star Rating Measure Cut Points. https://www. cms.gov/medicare/prescription-drug-coverage/ prescriptiondrugcovgenin/downloads/2014-trends-in-part-c-and-dstar-rating-measure-cut-points.Pdf. Accessed April 21, 2017.

25. Choudhry NK, Shrank WH, Levin RL, et al. Measuring concurrent adherence to multiple related medications. Am J Manag Care. 2009;15(7): 457-464.

26. Aronson AR. Effective mapping of biomedical text to the UMLS Metathesaurus: the MetaMap program. Proc AMIA Symp. 2001;17-21. D010001275 [pii]. https://www.ncbi.nlm.nih.gov/pubmed/11825149

27. Chapman WW, Bridewell W, Hanbury P, Cooper GF, Buchanan BG. A simple algorithm for identifying negated findings and diseases in discharge summaries. J Biomed Inform. 2001;34(5):301-310. https://doi. org/10.1006/jbin.2001.1029

28. Levey AS, Stevens LA, Schmid $\mathrm{CH}$, et al. A new equation to estimate glomerular filtration rate. Ann Intern Med. 2009;150(9):604-612. https://doi.org/10.7326/0003-4819-150-9-200905050-00006

29. Gagne JJ, Glynn RJ, Avorn J, Levin R, Schneeweiss S. A combined comorbidity score predicted mortality in elderly patients better than existing scores. J Clin Epidemiol. 2011;64(7):749-759. https://doi.org/ 10.1016/j.jclinepi.2010.10.004

30. Glickman ME, Rao SR, Schultz MR. False discovery rate control is a recommended alternative to Bonferroni-type adjustments in health studies. J Clin Epidemiol. 2014;67(8):850-857. https://doi.org/10. 1016/j.jclinepi.2014.03.012

31. Storey JD. The positive false discovery rate: a Bayesian interpretation and the q-value. Ann Stat. 2003;31(6):2013-2035.

32. Desgraupes B. Clustering Indices. https://cran.r-project.org/web/packages/clusterCrit/vignettes/clusterCrit.pdf. Published 2013. Accessed January 31, 2018.

33. Bass AJ, Dabney A, Robinson Maintainer John Storey DD. Q-value estimation for false discovery rate control. http://qvalue.princeton. edu. Published 2016.

\section{SUPPORTING INFORMATION}

Additional supporting information may be found online in the Supporting Information section at the end of the article.

How to cite this article: Singh $\mathrm{K}$, Choudhry NK, Krumme AA, et al. A concept-wide association study to identify potential risk factors for nonadherence among prevalent users of antihypertensives. Pharmacoepidemiol Drug Saf. 2019;28:1299-1308. https://doi.org/10.1002/pds.4850 